\title{
Miocardiopatía dilatada: a propósito de un caso
}

S. Berbel León, H. Iglesias Franco ${ }^{1}$ Especialistas en Medicina Familiar y Comunitaria. 'Tutor Extrahospitalario. Centro de Salud Pedro Laín Entralgo. Alcorcón, Madrid

\section{RESUMEN}

La miocardiopatía dilatada es una enfermedad primaria miocárdica que en la mayoría de los casos es de causa idiopática. El consumo de alcohol es la causa más frecuente de los casos de origen conocido.

A continuación presentamos el caso de un paciente con un consumo excesivo de alcohol que debutó con clínica de insuficiencia cardiaca.

Con este artículo queremos hacer hincapié en la importancia de la labor que el médico de Atención Primaria puede tener en el despistaje inicial de esta patología, así como en el seguimiento posterior del paciente para la identificación precoz de las complicaciones.

Palabras clave: Alcoholismo. Insuficiencia cardiaca. Miocardiopatía dilatada. Atención Primaria.
Dilated cardiomyopathy: a case review

\section{ABSTRACT}

Dilated cardiomyopathy is a primary cardiac disease which aetiology is usually unknown. The most frequent secondary cause of dilated cardiomyopathy is chronic alcohol consumption.

In the following article we are going to present you the case of a patient with an excessive consumption of alcohol that debuted with symptomatic congestive heart failure.

With this article we want to enhance the importance of Primary Health Care physician in the precocious identification of dilated cardiomyopathy, as well as in the patient's later pursuit for the precocious identification of the complications.

Key words: Alcoholism. Cardiac dysfunction. Dilated cardiomyopathy. Primary Health Care.

hipertensión, empleo de betaestimulantes o consumo de alcohol ${ }^{3}$ (Tabla I). Puesto que existe una gran variedad de alteraciones adquiridas o hereditarias que pueden causar dilatación y disfunción miocárdica, el diagnóstico diferencial entre las formas primarias y secundarias tiene implicaciones pronosticas y terapéuticas importantes.

En el caso de que la etiología sea el alcoholismo 


\section{Tabla I}

\begin{tabular}{ll}
\multicolumn{2}{c}{ ETIOLOGíA DE LA MIOCARDIOPATía DILATADA } \\
\hline Idiopática & La más frecuente \\
\hline Componente familiar & Asociación a antígeno DRW, \\
Formas de causa conocida 4 y/o DR 4 \\
Alcohólica: la más frecuente \\
de las secundarias \\
Vírica aguda ${ }^{12}$ \\
Enfermedad de Chagas \\
Toxoplasmosis \\
Toxicidad por antracinas, por \\
irradiación \\
Tirotoxicosis o mixedema \\
Enfermedades del colágeno \\
Distrofia muscular progresiva \\
Hemocromatosis \\
Sarcoidosis \\
Mucopolisacaridosis
\end{tabular}

crónico, el desarrollo de disfunción cardiaca está relacionado con la dosis total de etanol consumida a lo largo de la vida del paciente ${ }^{4}$. Por otro lado el consumo de alcohol también puede producir arritmias $^{1,3,5}$, por ejemplo: fibrilación auricular (FA) paroxística, que pueden descompensar un cuadro larvado de insuficiencia cardiaca.

La incidencia anual en Estados Unidos y en Europa varía entre 2 y 8 casos por 100.000 habitantes.

En el manejo de la miocardiopatía dilatada el médico de Atención Primaria tiene un papel importante en el despistaje inicial y en el seguimiento de la enfermedad, así como en el diagnóstico precoz de las complicaciones.

\section{CASO CLÍNICO}

Paciente de 52 años con traumatismos de repetición. No tiene historia de hipertensión, diabetes, ni dislipemia. Tampoco presenta alergias medicamentosas, ni consume fármacos habitualmente.

Enfermedad actual:

Acude a la consulta porque desde hace tres meses se encuentra muy triste, con pocos deseos de hacer cosas, astenia, insomnio y suspiros frecuentes.

Además refiere sensación de vacío en el estómago, anorexia y pérdida de peso de aproximadamente $3 \mathrm{~kg}$ no acompañado de cambios en el ritmo intestinal ni dolor epigástrico. El paciente 1o relaciona con estrés laboral.

Inicialmente se cataloga como un síndrome depresivo ansioso. Se pauta tratamiento antidepresivo (fluoxetina) y ansiolítico (bromazepam) y se solicita analítica general con hormonas tiroideas.
Once días después acude de nuevo a la consulta por empeoramiento de su sintomatología. Se le realiza una nueva anamnesis dirigida, describiéndonos que desde hace dos semanas presenta astenia intensa, disnea de moderados esfuerzos, ortopnea de dos almohadas, disminución de la diuresis y tumefacción en miembros inferiores. También refiere que por las noches ha presentado episodios de palpitaciones en varias ocasiones, no acompañándose de dolor torácico. Preguntado por el consumo de alcohol dice que bebe "lo normal", si bien al realizar un cuestionario de ingesta etílica se calcula que su consumo asciende a $295 \mathrm{~g} / \mathrm{sem}$.

En la exploración física se objetiva: tensión arterial 130/86, estado nutricional normal. AC: rítmico a $100 \mathrm{lpm}$, sin soplos ni extratonos. AP: crepitantes bibasales. Abdomen: hepatomegalia a cuatro traveses de dedo de reborde costal. EEII: edemas en ambas piernas con fóvea hasta rodillas.

En la analítica destaca: GOT: 52, GPT: 36, GGT: 97. El hemograma y el resto de bioquímica fueron normales.

En ECG realizado en la consulta únicamente se objetiva taquicardia sinusal y extrasístoles ventriculares frecuentes.

Ante la sospecha clínica de insuficiencia cardiaca se deriva al hospital para realización de radiografía de tórax urgente. En la radiografía se objetiva cardiomegalia y edema intersticial difuso, sugestivo de insuficiencia cardiaca congestiva.

Con esta sospecha diagnóstica se inicia tratamiento con diuréticos, se le cita para revisión en dos días y se le deriva a la consulta de Cardiología para estudio, recomendándole que si en algún momento presenta palpitaciones acuda al Centro de Salud o a urgencias del hospital.

A los dos días vuelve a la consulta. El paciente acude al hospital por palpitaciones. En el hospital se le realizan las siguientes pruebas complementarias: ECG: FA con respuesta ventricular a $150 \mathrm{lpm}$ con alteraciones inespecíficas de la repolarización y extrasístoles. ECO 2D-Doppler: derrame pericárdico global ligero. Dilatación ligera de las cuatro cavidades. AI $52 \mathrm{~mm}$ VI con severa hipoquinesia global. Disfunción severa biventricular. FEVI: $26 \%$. Dilatación de cava inferior y venas suprahepáticas. IT e IM moderadas (II/IV) PAP sistólica 45 $\mathrm{mmHg}$.

Se llega a la confirmación del diagnóstico de miocardiopatía dilatada en relación con abuso etílico, e intrahospitalariamente se realiza digitalización i.v., se pone tratamiento diurético (furosemida), amiodarona, IECA (enalapril) y se inicia anticoagulación oral (acenocumarol), siendo dado de alta para control por el Servicio de Arritmias y por su médico de Atención Primaria. 


\section{DISCUSIÓN}

La miocardiopatía dilatada no es una enfermedad infrecuente en la práctica clínica y precisa la realización de una valoración conjunta por Atención Primaria y especializada.

El médico de Atención Primaria es el receptor del paciente. La clínica inicial suele ser un cuadro de insuficiencia cardiaca de predominio izquierdo (es decir, de disnea, que según la gravedad, puede variar desde la forma más leve; la disnea de esfuerzo, pasando por la ortopnea, la disnea paroxística nocturna y en su estadio más grave el edema agudo de pulmón), que suele aparecer en una fase avanzada de la enfermedad. También se puede manifestar inicialmente (aunque con menor frecuencia) como angina de pecho, embolias sistémicas, fibrilación auricular o arritmias ventriculares ${ }^{1}$.

Según el síntoma con que debute el paciente hay que hacer el diagnóstico diferencial con cuadros tales como insuficiencia coronaria, prolapso mitral, bronquitis crónica, asma, hipertiroidismo, hiperparatiroidismo, feocromocitoma, ansiedad, etc...

En la consulta de Atención Primaria en un paciente de mediana edad con auscultación cardiaca normal, la causa más frecuente de disnea no cardiaca es la respiratoria. Ambas pueden ser difíciles de distinguir, más aún por que ambas pueden coexistir, en mayor o menor grado, en el mismo paciente. También la disnea puede ser una manifestación en pacientes con trastornos de angustia y ansiedad que muestran una alta incidencia de somatizaciones que pueden manifestarse como sensación de falta de aire, "ahogos", suspiros profundos o ganas de respirar profundo... Una vez más la historia clínica y la exploración se hacen necesarias. La anemia, para ocasionar disnea, ha de ser de instauración brusca y de cierta magnitud. De lo contrario, la adaptación a niveles bajos de hemoglobina $(\mathrm{Hb})$ en un sujeto con función cardiaca normal suele ser buena. Por último, también hay que tener en cuenta que las enfermedades del tiroides (hipertiroidismo) pueden producir disnea de esfuerzo, taquicardia acusada con/sin insuficiencia cardiaca, fibrilación auricular... En este caso hay una serie de síntomas (intolerancia al calor, mixedema, aumento del ritmo intestinal, hiperhidrosis) y signos (bocio difuso, soplo tiroideo, signos oculares, ginecomastia) que nos orientan hacia el diagnóstico de dicha patología, que confirmaremos por la analítica.

Otras causas posibles de disnea de instauración más progresiva en pacientes de mediana edad pueden ser, por ejemplo, la obesidad por la oposición de la grasa abdominal a la expansión diafragmática de los pulmones en la inspiración. La falta de entrenamiento físico, que rara vez es reconocida por los pacientes como causa de disnea, es también un motivo frecuente de consulta en Atención Primaria.
Una anamnesis minuciosa (obteniendo información sobre factores de riesgo de enfermedad cardiovascular, antecedentes de enfermedades sistémicas, así como el inicio de la clínica y el tiempo de evolución) y una buena exploración física, nos harán sospechar la existencia de insuficiencia cardiaca o de reconocer la insuficiencia cardiaca congestiva (ICC) en los estadios lo antes posible.

Con el fin de aumentar el valor predictivo de la clínica, ya que los síntomas y signos considerados aisladamente poseen insuficiente especificidad diagnóstica, éstos han sido agrupados en forma de criterios diagnósticos. Los más conocidos y utilizados son los de Framingham, que se exponen en la tabla II.

\begin{tabular}{|ll|}
\multicolumn{2}{c}{ Tabla II } \\
\cline { 1 - 2 } CRITERIOS DE FRAMINGHAM PARA EL DIAGNÓSTICO DE ICC \\
\hline Criterios mayores & Criterios menores \\
\hline Disnea paroxística nocturna & Disnea de esfuerzo \\
Estertores crepitantes & $\begin{array}{c}\text { Edemas en miembros inferio- } \\
\text { res }\end{array}$ \\
Edema agudo de pulmón & Derrame pleural \\
Cardiomegalia radiológica & Hepatomegalia \\
Tercer ruido cardiaco & Tos nocturna \\
Ingurgitación yugular & Taquicardia $>120$ lpm \\
Aumento de la presión venosa & \\
Reflujo hepatoyugular positivo & \\
Pérdida de peso $>4,5$ kg tras tto. & \\
\hline
\end{tabular}

Son necesarios para el diagnóstico la presencia de dos criterios mayores o bien uno mayor y dos menores. El criterio menor sólo es válido si se excluyen otras causas.

Cuando se sospecha por la anamnesis y exploración física de que estamos ante un cuadro de insuficiencia cardiaca se deben realizar de forma sistemática una serie de pruebas complementarias que nos permitan confirmar nuestro diagnóstico.

Se debe solicitar un electrocardiograma (ECG), una radiografía de tórax y una analítica, así como un ecocardiograma, que nos confirmará el diagnóstico.

El ECG es inespecífico, si bien puede aportar datos indirectos como pueden ser la existencia de hipertrofia de ventrículo izquierdo, isquemia o necrosis miocárdica, que pueden constituir la alteración orgánica que subyace en el corazón de un paciente que comienza a tener disnea. Otras alteraciones frecuentes en la IC son la taquicardia sinusal o la fibrilación auricular. La presencia de arritmias auriculares o ventriculares puede descompensar una IC, hasta entonces subclínica y el ECG aporta información indispensable para un trata- 
miento eficaz. La presencia de un ECG normal debe cuestionar el diagnóstico de IC, ya que posee un valor predictivo negativo muy elevado.

La radiografía de tórax aporta información sobre la morfología y tamaño de las cavidades cardiacas (especialmente aurícula izquierda: AI y ventrículo izquierdo: VI) y del grado de congestión pulmonar. Además permite descartar otras patologías y valorar posibles factores precipitantes (TEP, neumonía, etc.).

La analítica permite descartar o sospechar enfermedades concomitantes que pueden ser la causa de los síntomas o bien actuar como precipitantes (p.e. anemia, poliglobulia, insuficiencia renal, patología tiroidea). La valoración de la función hepática es necesaria para evaluar si existe repercusión de la IC sobre el hígado o enfermedades asociadas. $\mathrm{La}$ función renal debe conocerse y monitorizarse en relación con el tratamiento (IECA, diuréticos, digoxina, etc...).

El ecocardiograma es una prueba complementaria que no está disponible en Atención Primaria. Se considera imprescindible su realización en todo paciente con diagnóstico clínico fundado de IC, con la finalidad de confirmar el diagnóstico clínico y etiológico, y objetivar de una manera más precisa la función sistólica y diastólica ventricular. No disponer de esta prueba supone asumir la posibilidad tanto de falsos positivos como de falsos negativos en el diagnóstico, con lo que eso conlleva.

Los hallazgos característicos de la miocardiopatía dilatada en el ecocardiograma (independientemente de la etiología) son una reducción global de la contractilidad (aunque pueden identificarse defectos segmentarios), incremento de los diámetros y volúmenes ventriculares, dilatación auricular, aumento de la masa cardiaca total, disminución de la apertura de la válvula mitral $\mathrm{y}$, si existe un aumento del tamaño del corazón superior al $25 \%$, podemos encontrar insuficiencia mitral. Además se puede detectar la existencia de trombos de localización ventricular o auricular.

Es imprescindible la colaboración entre Atención Primaria y especializada para la realización de pruebas complementarias necesarias para el diagnóstico etiológico de la insuficiencia cardiaca. Inicialmente el ecocardiograma y, posteriormente, en caso de considerarse necesario, ventriculografía isotópica ${ }^{1}$ (valoración de metabolismo y perfusión de miocardio), ergometría (valoración objetiva de la capacidad funcional), Holter (en pacientes con arritmias o síncope), o cateterismo (en IC de origen isquémico, para valorar la posibilidad de revascularización).

En lo que se refiere al tratamiento de la IC en el seno de una miocardiopatía dilatada hay que tener en cuenta si la etiología de la enfermedad es conocida o desconocida, en cuyo caso carece de tratamiento específico ${ }^{1}$.
En caso de ser secundaria a alguna de las enfermedades citadas en la tabla I, se realizará un tratamiento etiológico, si la patología de base tiene tratamiento específico.

En el caso de nuestro paciente el consumo crónico de alcohol es la causa de la dilatación ventricular y, secundariamente, de la disfunción ventricular sistólica ${ }^{1,6}$. Por tanto, el primer escalón en su tratamiento es la abstinencia alcohólica ${ }^{1,3,6,7}$. Sólo un $50 \%$ de pacientes logran suprimir el consumo de alcohol de por vida. En caso de no alcanzar este objetivo, se ha comprobado en varios estudios que la reducción de la ingesta enólica a menos de 60 $\mathrm{g}$ /día mejora la evolución de los pacientes con disfunción ventricular ya establecida .

Es fundamental la labor del médico de Atención Primaria en la educación del paciente, en el control de los factores desencadenantes de la insuficiencia cardiaca, así como en el inicio del tratamiento precoz, ya sea con medidas no farmacológicas o farmacológicas, incluso cuando el paciente se encuentra asintomático ${ }^{8,9}$.

El seguimiento del tratamiento de la miocardiopatía dilatada en el caso de nuestro paciente consistiría en (Tabla III):

1. Tratamiento no farmacológico:

- Ayuda constante a la deshabituación etílica del paciente, ofertándole apoyo psicológico y/o psiquiátrico si fuera necesario ${ }^{5}$.

- Recomendar una ingesta calórica adecuada y comidas menos copiosas y más frecuentes.

- Restricción del consumo de sal a 2-3 g de $\mathrm{Na}^{+}$ al día en caso de IC establecida. En fases de descompensación reducción $\mathrm{a}<\mathrm{de} 1 \mathrm{~g}$ al día.

- Restricción hídrica en casos de hiponatremia dilucional o en descompensaciones importantes.

-Debe desaconsejarse el consumo de tabaco y de bebidas excitantes.

-En situaciones de desestabilización el reposo está indicado siempre, sin olvidar la prevención de enfermedad tromboembólica e intentando prevenir el decondicionamiento físico. En caso de enfermedad estable, el ejercicio físico regular mejora los síntomas, la capacidad funcional y la calidad de vida en los pacientes con IC.

Educar para detectar signos de alarma: ganancia de peso inexplicada, edemas, disminución de la cantidad de orina, empeoramiento del grado funcional, tos irritativa persistente (sobre todo nocturna), dolor torácico, disnea brusca o cansancio intenso.

-En estos pacientes se debe recomendar vacunación antigripal y antineumocócica, realizar un tratamiento precoz de las infecciones y de la fiebre.

-Es preciso evitar fármacos tales como los AINE, antiarrítmicos de clase I, antagonistas de los canales de calcio, antidepresivos tricíclicos, corticoides, litio, bicarbonato sódico o comprimidos efervescentes. 
2. Tratamiento farmacológico (Tabla I):

- Se debe comenzar con diuréticos a dosis altas y bajas de inhibidores de la enzima conversora de angiotensina (IECA) lo antes posible, y si es posible, comenzar de forma simultánea el tratamiento, e ir reduciendo y aumentando dosis respectivamente. Si existe contraindicación para IECA, se debe considerar la utilización de un antagonista de los receptores de angiotensina II (ARA II). En el caso de nuestro paciente sólo se inició tratamiento con diuréticos aunque lo más correcto habría sido iniciar el tratamiento con un IECA unido al diurético.

- Una vez estabilizado el paciente se debe introducir un betabloqueante (carvedilol, metoprolol, bisoprolol) en ausencia de contraindicaciones, comenzando con dosis muy bajas y con titulación cuidadosa.

-Espironolactona, en ausencia de contraindicaciones, a dosis bajas de $25 \mathrm{mg}$ /día. Se debe controlar la función renal y el $\mathrm{K}^{+}$.

-De forma individualizada se debe considerar la utilización de digoxina en caso de FA. La asociación de IECA y ARA II con el objetivo de control sintomático. Asociar diuréticos de diferente clase.

En caso de etiología isquémica considerar nitratos y en caso de hipertensión arterial (HTA/angina) considerar amlodipino.

- También es importante comenzar tratamiento anticoagulante ante circunstancias tales como IC

\section{Tabla III}

\section{TRATAMIENTO DE LA MOCARDIOPATÍA DILATADA}

\section{Prevención de la insuficiencia cardiaca}

Restricción de la actividad física y reposo

Pérdida de peso si el paciente es obeso ${ }^{8}$

Restricción dietética de sodio $(<2 \mathrm{~g} / \text { día })^{8}$

Control de FRCV: HTA, hiperlipidemia, DM $^{8}$

Tratamiento de patología tiroidea ${ }^{8}$

Tratamiento con IECA, que son los únicos fármacos que han demostrado prolongar la supervivencia y mejorar la calidad de vida del paciente $e^{8,9}$

Control de la frecuencia cardiaca en pacientes con taquiarritmias: digital ${ }^{8}$

Betabloqueantes en pacientes con IAM reciente sin alteración de FEVI 0 en pacientes con FEVI disminuida que no tengan antecedentes de $\mathrm{IAM}^{8}$

\section{Tratamiento de la insuficiencia cardiaca ${ }^{3}$}

Mantener las medidas para la prevención de insuficiencia cardiaca ${ }^{8}$

Tratamiento diurético en pacientes con retención de líquidos (tiazidas; si no responde: diuréticos de asa +/espironolactona) ${ }^{8}$

Interrupción de fármacos inotrópicos negativos y si se precisa administración de inotrópicos positivos ${ }^{8}$

Oxigenoterapia en caso de hipoxemia

FRCV: Factores de riesgo cardiovascular. HTA: Hipertensión arterial. DM: Diabetes Mellitus. IAM: Infarto agudo de miocardio. FEVI: Fracción de eyección de ventrículo izquierdo. aguda que requiera encamamiento, asociación con FA, episodios previos de embolia y si la FEVI < $30 \%$. Este tratamiento se puede instaurar tanto en Atención Primaria como en especializada, si no existe contraindicación y hay una colaboración continua entre ambos niveles. El INR que se debe mantener en estos pacientes debe estar entre 2 y 3.

Independientemente de los tratamientos farmacológicos, un seguimiento coordinado y cercano de los pacientes con IC, ha demostrado ofrecer un beneficio significativo a estos pacientes, reduciendo la posibilidad de reingreso hospitalario. En este contexto, el médico de familia tiene una posición privilegiada por su accesibilidad y conocimiento integral del paciente y su entorno.

Finalmente el médico de Atención Primaria debe tener claras las circunstancias en que se debe derivar al paciente a consulta especializada:

1. Cuando se diagnostica por primera vez (como aclaramos anteriormente), para confirmar el diagnóstico y filiar la etiología.

2. En las fases de descompensación, cuando la IC es refractaria al tratamiento o cuando no podamos realizar seguimiento y control del mismo.

3. Ante la presencia de síntomas severos: disnea severa en reposo, anasarca, isquemia miocárdica, arritmias, etc. o de enfermedades graves concomitantes como embolismo pulmonar, neumonía u otra infección grave intercurrente, hemorragia digestiva, empeoramiento significativo de la función renal, anemia severa, etc.

4. Para estudio de posible trasplante cardiaco: en menores de 65 años con IC intratable, si no se ha observado mejoría después de 3 meses de abstinencia etílica ${ }^{8,10}$ y si no existe contraindicación.

En definitiva, es preciso realizar un seguimiento coordinado multidisciplinar del paciente con IC y en su caso con miocardiopatía dilatada para un mejor control de dicha patología (Fig. 1).

\section{CONCLUSIONES}

1. Es fundamental confeccionar una historia clínica completa, así como una exploración física minuciosa en todos los pacientes y en todas las consultas que se realicen ${ }^{8}$. No haberlo hecho en nuestro paciente supuso un retraso en el diagnóstico.

2. Si un paciente tiene antecedentes de traumatismos de repetición hay que sospechar abuso etílico. Siempre hay que preguntar de forma dirigida la cantidad de alcohol consumida, haciendo un cálculo de la ingesta enólica ${ }^{8}$.

3. Ante un paciente con consumo crónico de alcohol hay que pensar en la posible existencia de disfunción miocárdica aunque el paciente se encuentre asintomático, y debemos comenzar con 


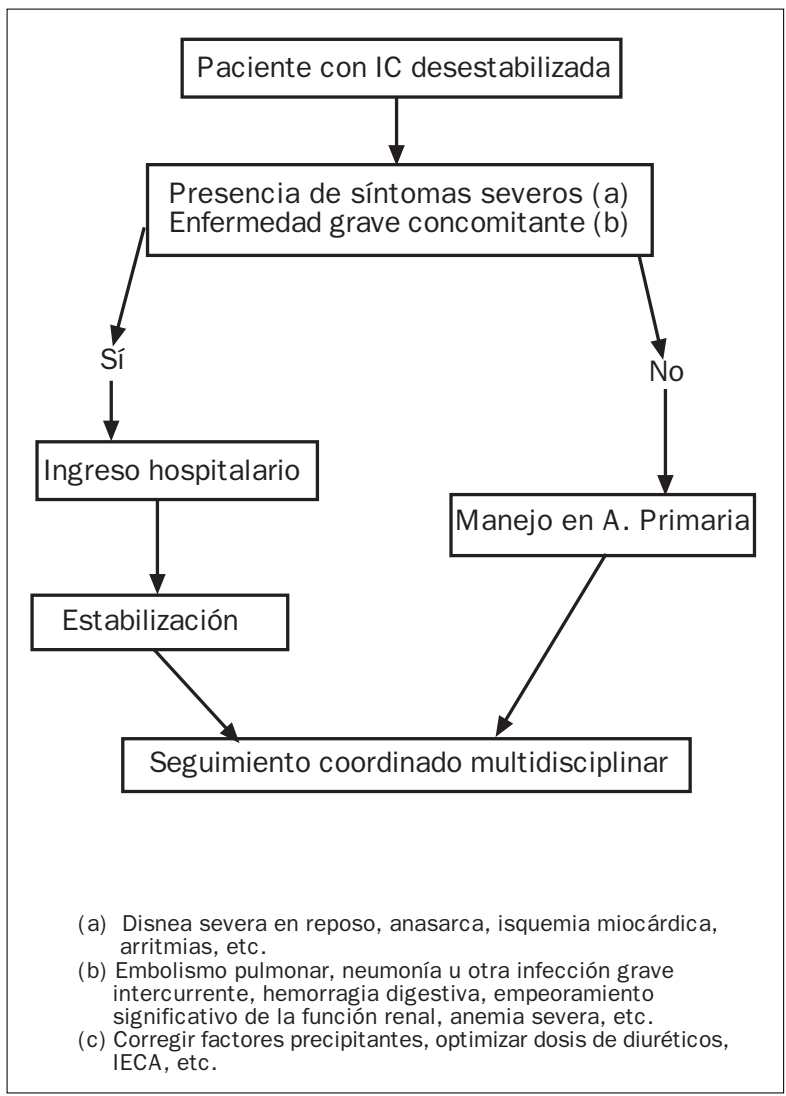

Figura 1

Manejo de la IC desestabilizada. medidas de soporte para lograr la abstinencia alcohólica o en caso de no conseguirse dicho objetivo reducir la ingesta etílica a menos de $60 \mathrm{~g} /$ día $^{1,3,5-7}$.

4. Ante un paciente con miocardiopatía dilatada se debe iniciar tratamiento con IECA incluso cuando el paciente esté asintomático, ya que estos fármacos han demostrado prolongar la supervivencia $^{8,9}$. En el caso de nuestro paciente sólo se inició tratamiento con diuréticos aunque habría sido más correcto iniciar con IECA unido al diurético.

5. El manejo del paciente con miocardiopatía dilatada debe ser conjunto entre Atención Primaria y Especializada. Debemos iniciar el tratamiento farmacológico en Atención Primaria y colaborar en el seguimiento del paciente, derivándole a consulta Especializada en caso de que aparezcan complicaciones no tratables ambulatoriamente (Fig. 1).

CORRESPONDENCIA:

Susana Berbel León

$\mathrm{C} /$ Venus, $13-2^{\circ} \mathrm{C}$

28925 San José de Valderas, Madrid

\section{Bibliografía}

1. Wynne J, Braunwald E. Miocardiopatías y Miocarditis. En: Harrison. Principios de Medicina Interna. $15^{\mathrm{a}}$ ed. Madrid: McGraw-Hill-Interamericana de España, 2002. p. 1598-604.

2. Navarro-López F. Miocardiopatías. En: Farreras. Medicina Interna. $14^{\mathrm{a}}$ ed. Madrid: Hartcourt, 2000. p. 656-7.

3. Schoppet M, Maisch B. Alcohol and the heart. Herz 2001; 26: $345-52$.

4. Fernández-Sola J, Nicolás JM, Pare JC, Sacanella E, Fatjo F, Cofan M, et al. Diastolic function impairment in alcoholics. Alcohol Clin Exp Res 2000; 24: 1830-5.

5. Spies CD, Sander M, Stangl K, Fernandez-Sola J, Preedy VR, Rubin E, et al. Effects of alcohol on the heart. Curr Opin Crit Care 2001; 7: 337-43.

6. Stollberger C, Finsterer J. Reversal of dilated to hypertrophic cardiomyopathy after alcohol abstinence. Clin Cardiol 1998; 21: 365-7.

7. Fauchier L, Babuty D, Poret P, Casset-Senon D, Autret ML, Cosnay $\mathrm{P}$, et al. Comparison of long-term outcome of alcoholic and idiopathic dilated cardiomyopathy. Eur Heart J 2000; 21: 267-9.
8. Hunt SA, Baker DW, Chin MH, Cinquegrani MP, Feldman AM, Francis GS, et al. ACC/AHA guidelines for the evaluation and management of chronic heart failure in the adult: executive summary: a report of the American College of Cardiology/American Heart Association Task Force on Practice Guidelines (Committee to Revise the 1995 Guidelines for the Evaluation and Management of Heart Failure). Circulation 2001; 104: 29963007.

9. Gavazzi A, De María R, Parolini M, Porcu M. Alcohol abuse and dilated cardiomyopathy in men. Am J Cardiol 2000; 85: 1114-8.

10. Coignard E, Blanchard B, Jault F, Dorent R, Vaissier E, Nataf $P$, et al. Alcoholic cardiomyopathy and heart transplantation. Arch Mal Coeur Vaiss 1998; 91: 45-51.

11. McKenna CJ, Codd MB, McCann HA, Sugrue DD. Alcohol consumption and idiopathic dilated cardiomyopathy: a case control study. Am Heart J 1998; 135: 833-7.

12. Constant J. The alcoholic cardiomyopathies-genuine and pseudo. Cardiology 1999; 91: 92-5. 